\title{
A CASE REPORT ON SERRATIA MARCESCENS
}

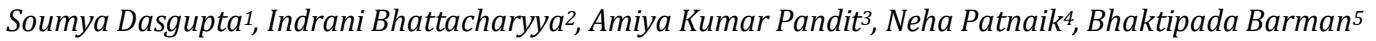

1 Postgraduate, Department of Microbiology, CSTM.

${ }^{2}$ Associate Professor, Department Microbiology, $\mathrm{CNMCH}$.

${ }^{3}$ Postgraduate, Department of Microbiology, CSTM.

${ }^{4}$ Postgraduate, Department of Microbiology, CSTM.

5Postgraduate, Department of Microbiology, CSTM.

\section{ABSTRACT}

Serratia marcescens is widely distributed in nature. Pigmented and non-pigmented strains are found from time to time in the human respiratory tract, faeces, urine, wounds and blood. Most infections are nosocomial. We isolated a pigmented Serratia marcescens strain from sputum of a patient who was admitted with fever and chest symptoms. Isolation of Serratia species as a nosocomial organism is an occasional finding. However, community acquired Serratia pneumonia is uncommon, hence we are reporting this case study.

\section{KEYWORDS}

Lower Respiratory Tract Infections, Serratia Marcescens, Pneumonia, Enterobacteriaceae, Sputum, Coccobacilli.

HOW TO CITE THIS ARTICLE: Dasgupta S, Bhattacharyya I, Pandit AK, et al. A case report on Serratia marcescens. J. Evolution Med. Dent. Sci. 2016;5(75):5603-5605, DOI: 10.14260/jemds/2016/1264

\section{INTRODUCTION}

Serratia marcescens is a member of the Enterobacteriaceae family. It is widely distributed in nature and can be isolated as saprophytes from soil and water. Reports of nosocomial infections caused by Serratia marcescens have increased in recent years. Infections include UTI, RTI, septicaemia, meningitis and endotoxic shock. Only small proportion $(<10 \%)$ of strains responsible for infection are pigmented. It is the most common clinical isolate among Serratia. ${ }^{1}$ We isolated Serratia marcescens from sputum of a hospitalised patient who was hospitalised with fever, cough and vomiting.

\section{PATIENT PROFILE}

A 43-year-old female, weighing $48 \mathrm{~kg}$, residing at Rajchandrapur, Howrah was admitted with complaints of high grade fever for last 15 days, cough, chill \& rigor. Patient had 2-3 episodes of vomiting initially, which later subsided. She had occasional body ache, pain abdomen and weakness. She was non-hypertensive and non-diabetic.

Initially, she was treated by a local physician with Tab Cefpodoxime; however, fever did not subside and she was admitted to Beliaghata ID \& BG hospital. There, she received Inj. Linezolid, Inj. Meropenem and Inj. Levofloxacin for 3 days. However, she was referred here for better diagnosis and management.

General examination was normal; however, on chest examination, left lower zone crepitations were heard. Other systemic examination was normal.

USG abdomen showed normal study. LFT-normal study, Hb-10.8 g, TLC-11400/cu. mm; N53 L45, HIV I \& HIV2 - Nonreactive, NS1 \& Dengue IgM non-reactive, Widal test was

Financial or Other, Competing Interest: None.

Submission 10-08-2016, Peer Review 03-09-2016,

Acceptance 09-09-2016, Published 19-09-2016.

Corresponding Author:

Dr. Indrani Bhattacharyya,

$37 \mathrm{~J} / 1$ D, Raja Manindra Road,

Kolkata-700037.

E-mail: indranichaudhuri@yahoo.com

DOI: $10.14260 /$ jemds/2016/1264

\section{(c) $(\mathbf{P})$}

negative, Routine stool \& urine examinations were normal and Blood culture showed no growth.

Sputum microscopy showed presence of plenty of pus cells and Gram-negative bacilli.

\section{MATERIALS AND METHODS}

Sputum was inoculated in MacConkey agar and blood agar plates and was incubated aerobically at $37^{\circ} \mathrm{C}$ for 24 hours. On MacConkey agar convex, effuse, colonies with irregular cremated edges developed with bright red pigmentation. On blood agar convex, raised, greyish colonies with narrow zone of haemolysis was seen.

Gram stain showed small Gram-negative coccobacilli. Hanging drop preparation showed motile organism.

\section{Biochemical Tests}

Indole- Neg; TSI-K/A, no H2S; Urease -Neg, Citrate +ve, Oxidase -Neg, Lysine +ve, Ornithine +ve, Arginine - Neg, PPA - Neg, Reduced nitrate to nitrite, ONPG +ve, Glucose- Acid, no gas; Sucrose-Acid, no gas; Mannitol-Acid, no gas; Arabinosenot fermented.

Morphology, colony characters and biochemical tests were indicative of Serratia marcescens. ${ }^{2,3}$ We inoculated two nutrient agar tubes and kept one of them in room temperature $\left(25^{\circ} \mathrm{C}\right.$ ) and another one at $37^{\circ} \mathrm{C}$ for pigment production. Abundant deep red non-diffusible pigment developed on nutrient agar kept in air at room temperature $\left(25^{\circ} \mathrm{C}\right)$. At $37^{\circ} \mathrm{C}$ there was less pigment production. The pigment was soluble in absolute alcohol, acetone, chloroform but insoluble in water. ${ }^{4}$

Based on Gram-stain, morphology, motility, colony character, biochemical tests, pigment production and character of the pigment, the organism is diagnosed as Serratia marcescens.

We also subcultured the organism and put it in Vitek2 which also diagnosed it as Serratia marcescens.

\section{Drug Susceptibility}

Done on Mueller-Hinton agar plate also shows non-diffusible red pigmentation and the following sensitivity patternSensitive to: Piperacillin-Tazobactam, Cotrimoxazole, Imipenem, Gentamicin, Ciprofloxacin and Cefixime. 
Resistant to Colistin, which also justified the diagnosis as Serratia are intrinsically resistant to Colistin. ${ }^{5}$

\section{TREATMENT HISTORY}

Patient was on IV fluid and Inj. Meropenem. Ciprofloxacin was added later. Patient was responding to treatment.

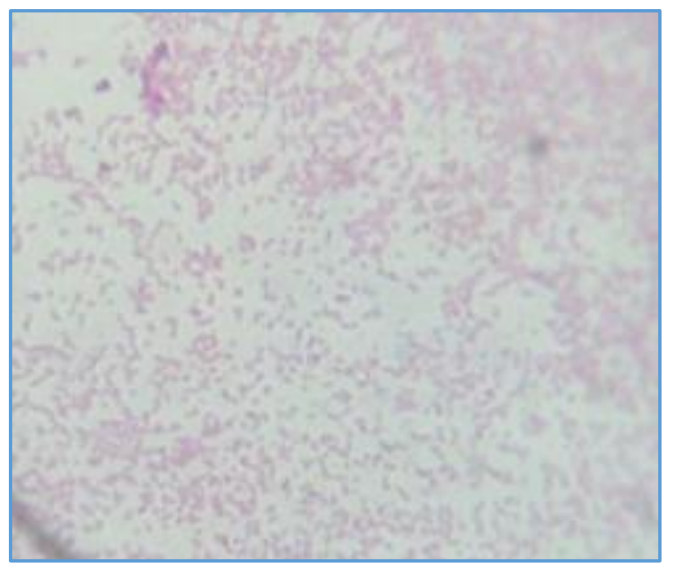

Fig. 1: Gram Stain

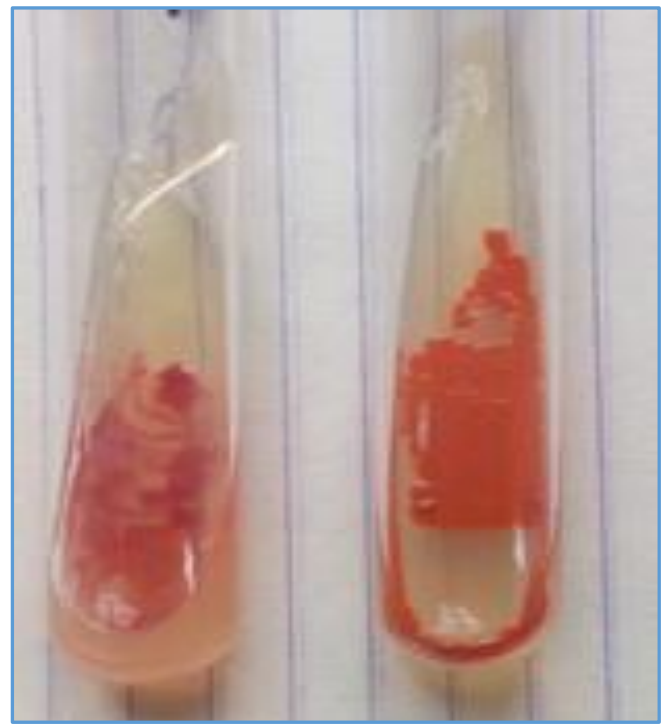

Fig. 2: Pigment Production Left-370 C; Right- $25^{\circ} \mathrm{C}$

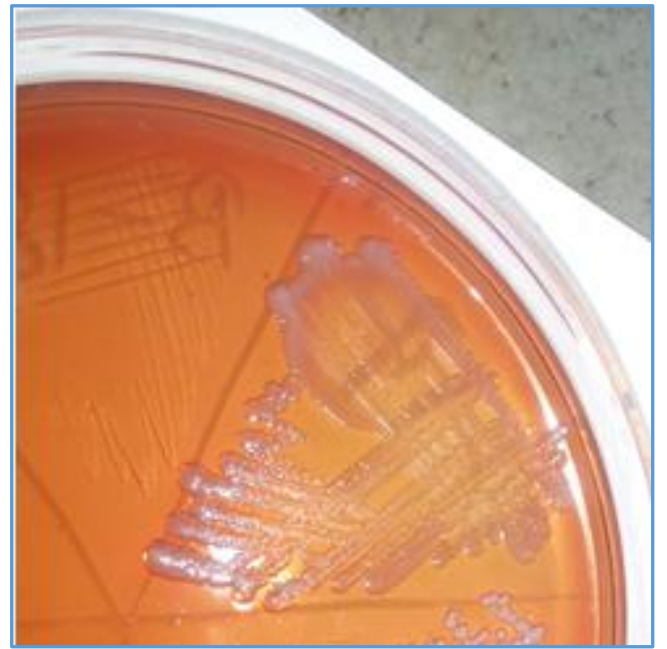

Fig. 3: Colony on Blood Agar

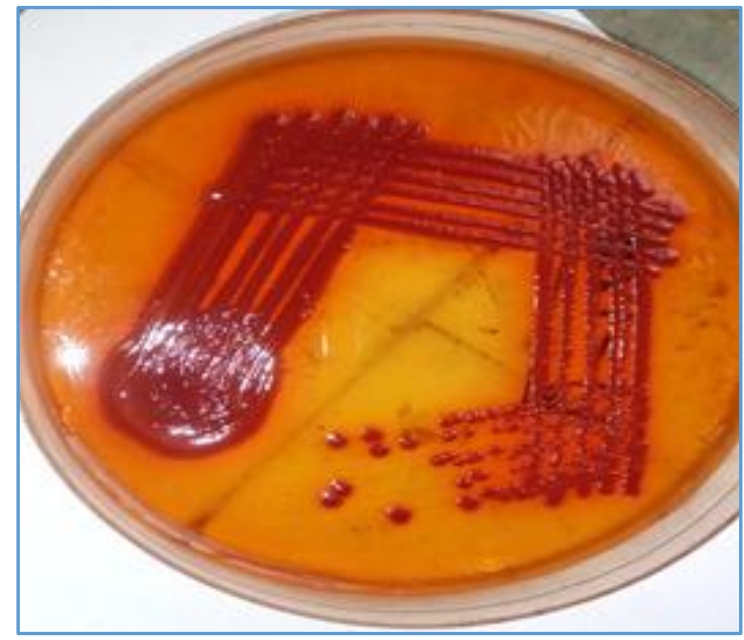

Fig. 4: Colony on MacConkey Agar

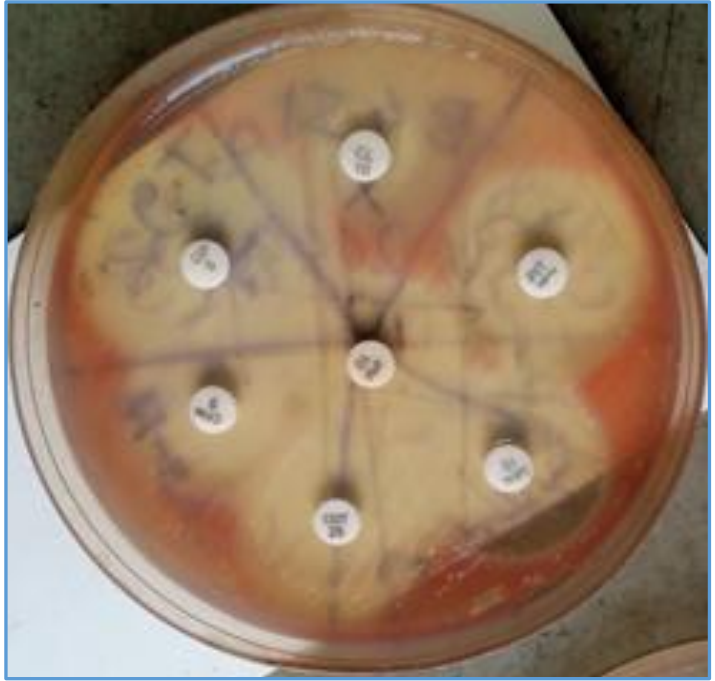

Fig. 5: Drug Susceptibility on Mueller-Hinton Agar with Disk diffusion method (CL-Colistin, PIT-PiperacillinTazobactam, GEN-Gentamicin, COT-Cotrimoxazole, CFMCefixime, CIP-Ciprofloxacin, IPM-Imipenem

\section{DISCUSSION}

Serratia marcescens was first described and named by Bizio in 1823. Gaughran (1969) assumed that the first recorded account of Serratia marcescens dates back to 332 B.C.

Genus Serratia contains 9 species. Most strains of S. plymuthica, S. rubidaea (syn. S marinorubra) and some strains of S. marcescens produce magenta red pigment (prodigiosin) but former two species are negative for lysine and ornithine decarboxylase. S. odorifera is a non-pigmented species which is lysine positive but has a characteristic musty potato like odour which aids its recognition in the laboratory. ${ }^{1}$ Some strains of S. marcescens may grow in chlorhexidine based disinfecting solution. Some of the strains are resistant to cephalosporins. Such resistance is frequently plasmid mediated and readily transferrable to other members of the Enterobacteriaceae. ${ }^{6}$

Nosocomial Serratia marcescens infection is not an uncommon finding. These infections pose the problem of drug resistance. Community acquired Serratia marcescens infection is not a very common finding as yet, but the problem of drug resistance is likely to occur considering the track record of the organism. As the drug resistance is 
transferable, the medical fraternity should be very cautious and make a concerted effort to contain such infections particularly when they are found in a community setting. Microbiologists and physicians together should see to it that the detected cases get completely cured and community spread is minimised. For this not only should we try for good drug compliance but we should also pay attention to proper health education so that spread of infection among relatives and contacts is prevented.

\section{REFERENCES}

1. Mackie and McCartney. Practical medical microbiology. $14^{\text {th }}$ edn. 2012:371.

2. Davis BR, Ewig WH, Reavis RW. The biochemical reaction given by members of the Serratia group. Int Bull Bacteriol Nomenclature \& Taxonomy 1957;7(4):151-60.
3. Difco laboratories: differentiation of Enterobacteriaceae by biochemical tests, Detroit 1980 .

4. Willium RP, Green JA, Rappaport DA. Studies on pigmentation of Serratia marcescens 1. Spectral and paper chromatographic properties of prodigiosin. J Bacteriol 1956;71(1):115-20.

5. CLSI Performance standards for antimicrobial susceptibility testing. $26^{\text {th }}$ edn. CLSI supplement M100S. Wayne, PA 2016:214.

6. Mandell GL, Bennett JE, Dolin R. Principles and practices of infectious diseases. $7^{\text {th }}$ edn. Philadelphia: Churchill Livingstone 2010. 